EPJ Web of Conferences 64, 05007 (2014)

DOI: $10.1051 /$ epjconf/ 20146405007

(C) Owned by the authors, published by EDP Sciences, 2014

\title{
Star/disk interaction and angular momentum evolution model for solar-like stars
}

\author{
Florian Gallet ${ }^{1, a}$ \\ ${ }^{1}$ UJF-Grenoble 1/CNRS-INSU, Institut de Planétologie et d'Astrophysique de Grenoble (IPAG) UMR 5274, \\ F-38041 Grenoble, France
}

\begin{abstract}
The magnetic field in young stellar object is undoubtedly the most important component when one dealing with the angular momentum evolution. It controls this latter one from the pre-main sequence, during the so-called disk locking phase where the stars magnetically interact with their surrounding disk, to the main-sequence through powerful stellar winds that remove angular momentum from the stellar surface. We present new models for the rotational evolution of solar-like stars between $1 \mathrm{Myr}$ and $10 \mathrm{Gyr}$ with the aim to reproduce the distributions of rotational periods observed for star forming regions and young open clusters within this age range. We based our simulation on a recent model dedicated to the study of the angular momentum evolution of solar-type stars. This model include a new wind braking law based on recent numerical simulations of magnetized stellar winds and a specific dynamo and mass-loss prescription are used to link the angular momentum loss-rate to angular velocity evolution. The model additionally allows for a core/envelope decoupling with an angular momentum transfer between these two regions. Since this former model didn't include any physical star/disk interaction description, two star/disk interaction processes are eventually added to it in order to reproduce the apparent small angular velocities to which the stellar surface is subject during the disk accretion phase. We have developed rotational evolution models for slow, median and fast rotators including two star/disk interaction scenarios that are the magnetospheric ejection and the accretion powered stellar winds processes. The models appear to fail at reproducing the rotational behaviour of solar-type stars except when a more intense magnetic field is used during the disk accretion phase.
\end{abstract}

\section{Introduction}

Classical T Tauri stars (CTTs) are very active pre-main sequence stars surrounded by an accretion disk $([1,2])$. The impact of their magnetic field on their dynamics and on the structure of their environment is very strong and lead to numerous physical phenomenon such as the truncation the disk and accretion of the material from it along funnel flows down to the stellar surface, launch of stellar winds along the opened magnetic field lines ([3-5]), and ejection of material because of magnetic star/disk interaction ([6-10]). The magnetic interaction between the star and its surrounding accretion disk remains one of the major unsolved issue concerning the angular momentum evolution of CTTs. It is now observationally clear (see [11-13]) that during the first $10 \mathrm{Myr}$ of their life the stars are

a e-mail: florian.gallet@obs.ujf-grenoble.fr

This is an Open Access article distributed under the terms of the Creative Commons Attribution License 2.0, which permits unrestricted use, distribution, and reproduction in any medium, provided the original work is properly cited. 
prone to held constant their rotation for several Myr $([14,15])$. Since CTTs accrete mass and angular momentum from their disk and they still contract during the pre-main sequence (PMS), they should spin-up in a few million years. Hence, this suggests that during this period a large fraction of the angular momentum is removed from the stars. Many theoretical advances have been made during the last years about the impact of the accretion/ejection phenomenon on the angular momentum evolution of young suns such as the magnetospheric ejection process (hereafter MEP, [10]) that describes the magnetic interaction between a stellar magnetosphere and an accretion disk, and the accretion power stellar wind (hereafter APSW, [3, 16, 17]) that predicts powerful stellar jets powered directly by the accreted material.

The aim here is to examine the impact of these different star/disk interaction scenarios on the angular velocity evolution and the conditions for which the surface angular velocity of the stars is held constant during the early PMS phase when the stars are still surrounded by an accretion disk. Since some previous works by [18] and [19] already studied such interaction we will focus on reproducing the rotational evolution of solar-like stars by comparing the models to the observations. This is the first time that these specific scenarios are incorporated into a "global" angular momentum evolution model which gives us the chance to analyse the impact of these interactions on the MS rotational behaviour.

\section{The model}

We use the model described in [15] that is dedicated to the study of the angular momentum evolution of solar-type star. Since this model didn't include any physical description of the star/disk interaction process we want to examine the impact of star/disk interaction scenarios on the early PMS angular momentum evolution. A more detailed description of the former model can be found in [15].

\subsection{Star/disk interaction}

For a few Myr during the early pre-main sequence, solar-type stars magnetically interact with their accretion disk. This star-disk magnetic coupling involves complex angular momentum exchange between the components of the system, including the accretion disk, the central star, and possibly both stellar and disk winds. Recently, accretion-powered stellar winds have been proposed as a way to remove from the central star the excess of angular momentum gained from disk accretion $[3,16,17]$. However, [20] showed that mass and energy supplied by accretion may not be sufficient to provide an efficient spin-down torque by accretion-driven winds. [10] proposed that magnetospheric reconnection events occurring between the star and the disk lead to ejection episodes that remove the excess angular momentum. Hence, a free parameter of the models is the accretion disk's lifetime, $\tau_{\text {disk }}$, i.e., the duration over which the star/disk interaction occur. After a time $\tau_{\text {disk }}$, the star is released from its disk, and is only subjected to angular momentum loss due to magnetized stellar winds (see below). Note that during most of the pre-main sequence, once the disk has been dissipated, angular momentum losses due to magnetized stellar winds are however unable to prevent the star from spinning up as its moment of inertia rapidly decreases towards the ZAMS (cf. [15, 21, 22]).

\subsubsection{Magnetospheric ejection}

The Magnetospheric Ejection process ([10]) results from the interaction of a stellar magnetosphere and an accretion disk that will produce the expansion and reconnection of the field lines connecting the star with its surrounding disk. The resulting inflation at mid-latitudes of the dipolar field lines is very 
dynamic and it is accompanied by outflows that could extract mass, energy, and angular momentum both from the disk and the star. Because of the magnetic field lines reconnection, these outflows detach from the magnetosphere and continue their propagation as magnetic islands disconnected from the central part of the star/disk system, in between the open magnetic surfaces anchored into the star and those anchored into the disk (see fig. 2 from [10]). Additionally the disk will be truncated and accretion curtains will form. The truncation radius $R_{t}$ is given by ([23])

$$
R_{t}=K_{t}\left(\frac{B_{p}^{4} R_{*}^{12}}{G M_{*} \dot{M}_{a c c}^{2}}\right)^{1 / 7},
$$

where $\dot{M}_{a c c}$ is the mass accretion rate and $K_{t}=0.5$ a normalisation constant. This region is connected within the Keplerian corotation radius

$$
R_{c o}=\left(\frac{G M_{*}}{\Omega_{*}^{2}}\right)^{1 / 3},
$$

so that, beyond this radius, the disk and the star do not have a direct magnetic connection. Because of the magnetic reconnection process the dipolar field lines will inflate at mid-latitudes resulting in the production of magnetospheric ejections (hereafter MEs).

We found that the mean magnetic field is not strong enough to held constant the surface angular velocity of the stars during the disk accretion phase. Thus, an another component of the magnetic field have to be use during the disk's lifetime to reproduce the observations. As a consequence we simply imposed a more intense magnetic field $B_{\text {mod }}$ during the disk accretion phase to produce a constant angular velocity. While the nature of this magnetic field is very well known from a theoretical point of view and it is associated to the dipolar component of the magnetic field, from an "observational" point of view this is not as clear since the real stellar magnetic field is clearly not a perfect dipole. Because of this issue we will not discuss the origin of this intense magnetic field. However, the strong values of $B_{\text {mod }}$ that is required to fit the observations can be considered as a preliminary result of the star/disk interaction process and only the order of magnitude of the magnetic field strength have to be taken into account.

A reasonably approximation for the spin-up torque due to accretion is

$$
\tau_{a c c}=K_{a c c} \dot{M}_{a c c} \sqrt{G M_{*} R_{t}}
$$

which is proportional to the mass accretion rate $\dot{M}_{a c c}$ and to the disk specific Keplerian angular momentum in the truncation region. As it has been shown in [10], one effect of the MEs is to reduce the "Keplerian" accretion torque. This is qualitatively expressed by the constant $K_{a c c}=0.4$ that translates the fact that a fraction $1-K_{a c c}$ of $\dot{M}_{a c c}$ is launched in the form of MEs. The torque exerted by stellar magnetic field lines coupled to a region of size $\Delta R$ of the MEs can be expressed as $([24,25])$

$$
\tau_{\text {mag }}=q \Delta R \frac{B_{d i p}^{2} R_{*}^{6}}{R_{t}^{4}},
$$

where $q=B_{\phi} / B_{z} \approx\left[\Omega_{M E s}-\Omega_{*}\right] / \Omega_{M E s}([16,24,26])$ is related to the differential rotation between the star and the MEs and can be expressed as

$$
q \propto K_{r o t}-\left(\frac{R_{t}}{R_{c o}}\right)^{3 / 2} .
$$


The $K_{\text {rot }}=0.7$ factor expresses the fact that the MEs rotate at a sub-Keplerian rate. Assuming $\Delta R \propto R_{t}$, the MEs torque can be written as:

$$
\tau_{M E}=K_{M E} \frac{B_{d i p}^{2} R_{*}^{6}}{R_{t}^{3}}\left[K_{\text {rot }}-\left(\frac{R_{t}}{R_{c o}}\right)^{3 / 2}\right]
$$

where $K_{M E}=0.21$. Notice that this torque spins-down the star only for $R_{t} / R_{c o}>K_{\text {rot }}^{2 / 3} \approx 0.8$ while for lower truncation radius the MEs spin-up the stellar surface.

\subsubsection{Accretion powered stellar winds}

During the T Tauri phase the stars are subject to numerous mechanisms: stellar contraction, accretion process, stellar winds, and magnetic star/disk interaction. The total torque applied on the stellar surface is the sum of all these contributions but since the angular momentum problem is a complex issue to tackle, one solution is to compare one spin-up torque to one spin-down torque. This section presents the accretion powered stellar wind process developed by [16] which proposed that the energy driving the stellar wind comes directly from the accretion power and that we can link the mass-loss rate to the mass-accretion rate

$$
\dot{M}_{\text {wind }}=Q_{a c c} \dot{M}_{a c c},
$$

where $Q_{a c c}$ is a proportionality constant set between $1 \%$ (low mass loss rate) to $10 \%$ (high mass loss rate). The latter one is the value adopted by $[3,16,17]$ which represents very powerful outflows.

For the mass accretion rate we assumed $\dot{M}_{a c c}=10^{-9} M_{\odot} / y r$ at $1 \mathrm{Myr}([2,27])$ and used a simple decay function based on [28] (i.e. $\dot{M}_{a c c} \propto t^{-1.2}$ )

$$
f_{\text {decay }}=\left(\frac{t_{\text {disk }}}{t_{\text {init }}}-1\right)^{-1.2}\left(\frac{t_{\text {disk }}}{t_{\text {star }}}-1\right)^{1.2},
$$

to express the evolution of the mass accretion rate

$$
\dot{M}_{a c c}=\dot{M}_{a c c, i n i t} f_{d e c a y},
$$

where $t_{\text {init }}$ is the starting age of our simulation, $t_{\text {disk }}$ the disk lifetime, and $t_{\text {star }}$ the age of the star. The characteristic of this decay function is to be equal to zero when $t_{\text {star }}=t_{\text {disk }}$ while an exponential decay will produce a non-zero accretion rate even after the disk dissipation.

\section{Spin equilibrium}

The free parameters of the model are the initial rotation period $P_{\text {init }}$ at $1 \mathrm{Myr}$, the core-envelope coupling time-scale $\tau_{c-e}$, the disk lifetime $\tau_{d i s k}$, and the calibration constant of the wind braking law $K_{1}$. This study adopts the same parametrization as described in [15]. Additionally, two free parameters are added here: the mass loss rate efficiency constant $Q_{a c c}$, and the strength of the magnetic field $B_{\text {mod }}$. We computed the angular velocity evolution of the fast, median, and slow rotator models, and examine the impact of the two star/disk interaction mechanisms on this evolution. Figure 1 shows the angular velocity evolution of the fast, median, and slow rotator models, respectively in blue, green, and red.

For the fast rotator model a disk's lifetime of $2.5 \mathrm{Myr}$ is used that lead to a rapid increase of the surface angular velocity of the stars during the PMS. The quite low initial rotation period $P_{\text {init }}=1.4 \mathrm{~d}$ is dictated by the fast rotation rate exhibited in the Orion Nebulae Cluster at $1 \mathrm{Myr}$ and the NGC 6530 cluster at 1.65 Myr. To fit the observational constraints from the PMS to the ZAMS a relatively short 


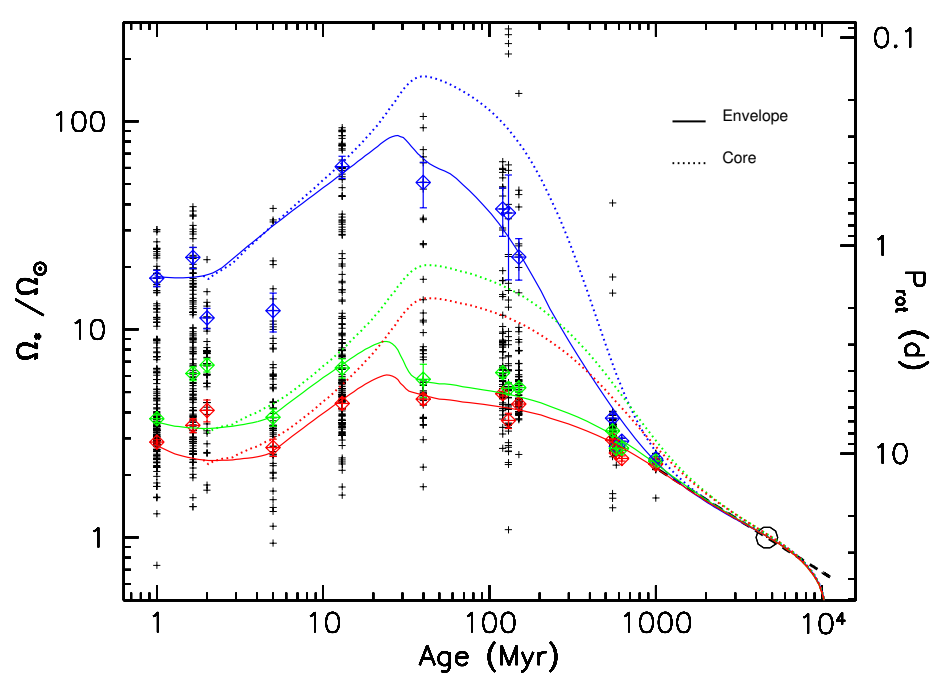

Figure 1. Angular velocity of the radiative core (dashed lines) and of the convective envelope (solid lines) is shown as a function of time for fast (blue), median (green), and slow (red) rotator models in the case $Q_{a c c}=1 \%$. The angular velocity is scaled to the angular velocity of the present Sun. The blue, red and green tilted square and associated error bars represent the 90th percentile, the 25th percentile, and the median, respectively, of the rotational distributions of solar-type stars in star forming regions and young open clusters obtained with a rejection sampling method (see [15]). The open circle is the angular velocity of the present Sun and the dashed black line illustrates the Skumanich's relationship, $\Omega_{*} \propto t^{-1 / 2}$.

core/envelope coupling timescale of $10-15 \mathrm{Myr}$ is needed. To reproduce the early-PMS observations we numerically set $B_{\text {mod }}=1000 \mathrm{G}$.

For the median and slow rotator models the initial rotation periods are 7 and $9 \mathrm{~d}$, respectively, as fixed by the rotation period distributions of the youngest PMS clusters. For both models we chose a disk lifetime of 5 Myr to reproduce the late PMS clusters. To account for the moderate velocities on the ZAMS, we had to assume a much longer core-envelope coupling timescale than for fast rotators, namely 28 and $30 \mathrm{Myr}$ for median and slow rotator models, respectively. The slow decline of surface rotation is due to angular momentum of the core resurfacing at the stellar surface on a timescale of $\simeq 100 \mathrm{Myr}$ in slow and moderate rotators and it accounts for the observed evolution of the lower envelope of the rotational distributions of early MS clusters. To reproduce the observations in these cases, $B_{\text {mod }}$ is set at 1600 and $1300 \mathrm{G}$ for the slow and median rotator models, respectively.

\section{Conclusion}

We combined two star/disk interaction scenarios in the "global" angular momentum evolution model described in [15] with the aim to study the angular velocity evolution of solar-like stars during the early-PMS phase. We found that the models fail to reproduce the observations except when a more intense magnetic field is used during the disk accretion phase. In presence of weak magnetic field the torque applied by the accretion disk on its star is not sufficient enough to efficiently extract angular momentum from the stellar surface to compensate the stellar contraction that otherwise dominate this evolution. These results are certainly not new since the star/disk interaction process is not a modern 
issue and that it has already been studied in the literature through different mechanisms (e.g. [18, 19]). The major difference of this study is that the resulting angular velocity evolution produces by the star/disk interaction is directly compare to the observations. However, this is a preliminary result and a more physical model is still to be developed especially in studying the impact of a non-axisymmetric and multipolar magnetic field on the angular velocity evolution.

\section{References}

[1] S. Edwards, P. Hartigan, L. Ghandour, C. Andrulis, aj 108, 1056 (1994)

[2] L. Hartmann, N. Calvet, E. Gullbring, P. D’ Alessio, apj 495, 385 (1998)

[3] S. Matt, R.E. Pudritz, apj 678, 1109 (2008), 0801.0436

[4] S.P. Matt, K.B. MacGregor, M.H. Pinsonneault, T.P. Greene, apj1 754, L26 (2012), 1206.2354

[5] S.R. Cranmer, S.H. Saar, apj 741, 54 (2011), 1108.4369

[6] F. Shu, J. Najita, E. Ostriker, F. Wilkin, S. Ruden, S. Lizano, apj 429, 781 (1994)

[7] J. Ferreira, G. Pelletier, S. Appl, mnras 312, 387 (2000)

[8] M.M. Romanova, G.V. Ustyugova, A.V. Koldoba, R.V.E. Lovelace, mnras 399, 1802 (2009)

[9] C. Zanni, J. Ferreira, aap 508, 1117 (2009)

[10] C. Zanni, J. Ferreira, aap 550, A99 (2013), 1211.4844

[11] S. Edwards, S.E. Strom, P. Hartigan, K.M. Strom, L.A. Hillenbrand, W. Herbst, J. Attridge, K.M. Merrill, R. Probst, I. Gatley, aj 106, 372 (1993)

[12] J. Bouvier, S. Cabrit, M. Fernandez, E.L. Martin, J.M. Matthews, aap 272, 176 (1993)

[13] L.M. Rebull, S.C. Wolff, S.E. Strom, aj 127, 1029 (2004)

[14] J. Irwin, J. Bouvier, The rotational evolution of low-mass stars, in IAU Symposium, edited by E. E. Mamajek, D. R. Soderblom, \& R. F. G. Wyse (2009), Vol. 258 of IAU Symposium, pp. 363-374

[15] F. Gallet, J. Bouvier, aap 556, A36 (2013), 1306.2130

[16] S. Matt, R.E. Pudritz, apjl 632, L135 (2005), arXiv : astro-ph/0510060

[17] S. Matt, R.E. Pudritz, apj 681, 391 (2008), 0801.0440

[18] A. Collier Cameron, C.G. Campbell, H. Quaintrell, aap 298, 133 (1995)

[19] S.P. Matt, G. Pinzón, R. de la Reza, T.P. Greene, apj 714, 989 (2010), 1005.0863

[20] C. Zanni, J. Ferreira, apj1 727, L22+ (2011), 1012 . 3333

[21] J. Bouvier, M. Forestini, S. Allain, aap 326, 1023 (1997)

[22] S. Matt, R.E. Pudritz, The nature of stellar winds in the star-disk interaction, in IAU Symposium, edited by J. Bouvier, I. Appenzeller (2007), Vol. 243 of IAU Symposium, pp. 299-306, 0707.0306

[23] N. Bessolaz, C. Zanni, J. Ferreira, R. Keppens, J. Bouvier, aap 478, 155 (2008), 0712 . 2921

[24] P.J. Armitage, C.J. Clarke, mnras 280, 458 (1996), arXiv: astro-ph/9512018

[25] S. Matt, R.E. Pudritz, mnras 356, 167 (2005), arXiv: astro-ph/0409701

[26] M. Livio, J.E. Pringle, mnras 259, 23P (1992)

[27] J. Muzerolle, L. Hartmann, N. Calvet, aj 116, 2965 (1998)

[28] A. Caratti O Garatti, R. Garcia Lopez, S. Antoniucci, B. Nisini, T. Giannini, J. Eislöffel, T.P. Ray, D. Lorenzetti, S. Cabrit, aap 538, A64 (2012) 